\title{
Polarization-induced distortion of ions in the pores of carbon electrodes for capacitors
}

\author{
C.O. Ania ${ }^{1,2}$, J. Pernak ${ }^{3}$, F. Stefaniak ${ }^{3}$, E. Raymundo-Piñero ${ }^{1}$, F. Béguin ${ }^{1 *}$ \\ ${ }^{1}$ CRMD, CNRS-Université, 1 B rue de la Férollerie, 45071, Orléans, France \\ ${ }^{2}$ Instituto Nacional del Carbón, INCAR-CSIC, Apdo. 73, 33080 Oviedo, Spain \\ ${ }^{3}$ Poznań University of Technology, Department of Chemical Technology, pl. Skłodowskiej- \\ Curie 2, 60-965 Poznań, Poland
}

\begin{abstract}
This paper reports the effect of confining ionic species of the electrolyte inside the porosity of carbon electrodes during the performance of electrochemical capacitors. Solvent-free ionic liquids and a conventional organic medium were used as electrolytes, while two series of carbons with controlled pore sizes - one of them obtained from nanocasting procedure - were used as electrode materials. Our results demonstrate that under the effect of the electric field applied during the polarization of the electrodes, desolvated ions enter distorted in pores smaller than their limiting size. In such conditions of confinement, it is demonstrated that the traditional concept of an electric double-layer formed by solvated ions adsorbed on both pore walls does not apply for sub-nanometer size pores.
\end{abstract}

\section{Introduction}

Electrical double layer capacitors (EDLC) are usually based on highly microporous carbon electrodes, because they are cheap and they can be produced with large pore volumes, enabling high values of capacitance [1,2]. However, it has been demonstrated that not all pores are effective in the charge accumulation due to molecular sieving effects and that a good matching between the electrode pore size and the dimensions of the ionic species is critical for an optimal performance of these systems [3-9]. Hence it is of paramount importance to clarify the pore-toion interactions in EDLC; for this purpose, the effective dimensions of the ionic species of the electrolyte should be accurately determined, as well as the dimensions of the pores in the electrode material. Because of the solvation of ions, the conventional electrolytes are not fully adapted for this approach.

In a previous work [3], we have demonstrated the sieving effect of a carbon host in the trapping of ions, by using solvent-free ionic liquids (ILs) as in situ probes to directly explore the accessibility of the ionic species to the nanotexture of a conventional activated carbon used as 
electrode material. Briefly, the results pointed out that when using conventional porous carbons -which are characterized by broad pore size distributions (PSD)-, very often a large fraction of the overall porosity is underused from an electrochemical point of view. It was therein suggested that rather than to report on an optimum pore size of an electrode material for electrochemical capacitors, the size of ions has to be taken into account, and thus the porosity of the carbon should be tailored to match the dimensions of the electrolyte chosen. Similar results on the relation between the ion dimensions and the pore size in EDLC have been further reported in the literature $[6,9]$.

However, the behaviour of ions in the confined space of nanopores as well as its effect on the performance of EDLC still remains not fully understood. Therefore, the aim of this paper was to investigate the capacitance response of supercapacitors and the pore-to-ion interaction from a two-fold approach: i) using pore-controlled carbons which possess narrow distribution of pore sizes as electrode materials; and ii) solvent-free ionic liquids of increasing sizes and tetraethylammonium tetrafluoroborate in acetonitrile as electrolytes.

As electrode materials we have used two pore-controlled carbons, one commercially available and the other in-house prepared from nanocasting procedures. As described in the literature, nanocasting has been considered as a unique and versatile method for providing well-designed and almost full pore-controlled carbon materials [10]. The resulting carbons possess a unique combination of high porosity, a controlled narrow distribution of pore size, which can be perfectly adapted to the size of the electrolyte, and straight channels enhancing the diffusion of ions. These features cannot be attained by conventional preparation processes (i.e, physical and chemical activation procedures).

Regarding the solvent-free electrolytes, we have selected a series of phosphonium-derived ILs featuring the same anion and different cations with increasing alkyl chain lengths. Due to the large electrochemical stability window of ILs, there has been a tremendous interest in their use as electrolytes in carbon-based electrochemical capacitors, being a strategy to develop high voltage devices - consequently high energy density ones [11]. In this sense, promising results have been reported so far, particularly for capacitors operating above room temperature where viscosity of ILs is decreased and conductivity is enhanced [12-15]. Notwithstanding the high

reachable voltage with ILs, our goal was not to obtain high energy devices but to profit from the ionic nature of ILs in the absence of solvents to use them as in situ probes to explore directly the accessibility of ions into the carbon porous network during the operation of an electrochemical capacitor (EC). Therefore, all our experiments have been realized by limiting the voltage to $1 \mathrm{~V}$ in order to avoiding any occurrence of ions intercalation, which might take place in carbon materials (porous and non-porous) at voltages higher than $2.5 \mathrm{~V}$ [16-18]. Indeed, intercalation processes could complicate the understanding of confinement effects. 
By using the two series of carbons along with ILs and a conventional electrolyte, it was possible to demonstrate that under the effect of polarization, the cations penetrate desolvated and distorted in the pores.

\section{Experimental}

\subsection{Materials}

Details on the preparation of the template carbon have been reported elsewhere [19]. Briefly, the synthesis is based the impregnation of an inorganic framework ( $\mathrm{NaY}$ zeolite) with furfuryl alcohol, followed by in-situ polymerization and a second step of chemical vapour deposition (CVD). After the impregnation, the precursor/zeolite composite was heated up to $700^{\circ} \mathrm{C}$ under nitrogen atmosphere, and then CVD was performed with propylene $\left(2 \%\right.$ in $\left.\mathrm{N}_{2}\right)$ for 4 hours at a flow rate of $150 \mathrm{ml} \mathrm{min}^{-1}$. The sample was further stabilized at $900^{\circ} \mathrm{C}$ for 3 hours under nitrogen. The silica matrix was etched using hydrofluoric acid (48\%) at room temperature, and the resulting template carbon was washed with concentrated $\mathrm{HCl}$ at $60^{\circ} \mathrm{C}$ and distilled water to remove traces of insoluble fluoride salts. The dried product is labeled Z4. A commercially available activated carbon cloth (CC) supplied by ACTITEX (France) was also used for manufacturing the electrodes. The advantage of the latter is that it can be used as self-supported electrode without the addition of binder and percolator.

\subsection{Ionic liquids}

ILs may be finely-tuned for an intended application by an adequate combination of the anion and the cation. In this study, we have synthesized phosphonium-derived ILs, $\left[\left(\mathrm{C}_{6} \mathrm{H}_{13}\right)_{3} \mathrm{P}\left(\mathrm{ROCH}_{2}\right)\right]\left[\mathrm{Tf}_{2} \mathrm{~N}\right]$, with increasing the cationic dimensions, following the procedure described elsewhere [20]. In the formula, $\mathrm{R}$ stands for the different alkyl chains and $\mathrm{Tf}_{2} \mathrm{~N}$ for the bis(trifluoromethanesulfonyl)imid counteranion. The ILs will be referred to in the text as IL-3, IL-4, IL-7 and IL-12 for R = propyl, butyl, heptyl and dodecyl, respectively. For clarity, the semi-structural drawings of the ILs are presented in Scheme 1. The physico-chemical characteristics and dimensions of the ILs have been reported elsewhere [3, 20].

$$
\mathrm{R}=\mathrm{C}_{3} \mathrm{H}_{7}(\mathrm{IL}-3) ; \mathrm{C}_{4} \mathrm{H}_{9}(\mathrm{IL}-4) ; \mathrm{C}_{7} \mathrm{H}_{15}(\mathrm{IL}-7) ; \mathrm{C}_{12} \mathrm{H}_{25}(\mathrm{IL}-12)
$$

Scheme 1. Semi-structural drawings of the ionic liquids used in this work. 


\subsection{Nanotextural and chemical characterization of carbons}

The nanotexture of carbons was characterized by $\mathrm{N}_{2}$ adsorption at $-196^{\circ} \mathrm{C}$ (Autosorb-1, Quantachrome). Before the experiments, the samples were outgassed under vacuum at $250{ }^{\circ} \mathrm{C}$ overnight. The isotherms were used to calculate the total pore volume, $\mathrm{V}_{\mathrm{T}}$, specific surface area, and pore size distribution using the quenched solid density functional theory (QSDFT) [21]. To avoid the inaccuracy of the BET method in the determination of the specific surface area in highly porous materials, this value was also calculated using the regularized DFT method $\left(\mathrm{S}_{\mathrm{DFT}}\right)$. The $\mathrm{V}_{\text {micro }} / \mathrm{V}_{\text {meso }}$ ratio was used to assess the relative contribution of micropores to mesopores in the overall pore volume.

\subsection{Electrochemical characteristics}

The electrodes are in the form of $1 \mathrm{~cm}^{2}$ self standing cloth disks or pellets obtained by pressing a mixture of template carbon material (85 wt. \%), percolator (Pure Black, Superior Graphite, 5 wt. \%) and polyvinylidene fluoride (PVDF, 10 wt. \%). Teflon Swagelock ${ }^{\circledR}$ cells constituted of two carbon electrodes of comparable mass (10 to $15 \mathrm{mg}$ ) electrically isolated by a glassy fibrous separator, were built in a glove-box under argon. In some experiments, the performance of the negative and positive electrodes could be recorded simultaneously to the operation of the capacitor by using a silver wire pseudo reference electrode. As the objective of this research was not to enhance the energy, electrochemical measurements were determined in a restricted voltage range - up to $1 \mathrm{~V}$ - and at a low scan rate of $1 \mathrm{mV} \mathrm{s}^{-1}$ using a VMP (Biologic, France) multichannel generator. This allows any kinetic restrictions imposed by the viscosity of the ionic liquids to be disregarded. For comparison purposes, an organic electrolytic solution -1 $\mathrm{mol} \mathrm{L}^{-1} \mathrm{TEABF}_{4}$ (tetraethylammonium tetrafluoroborate) in acetonitrile- has also been applied.

\section{Results and discussion}

\subsection{Dimensions of the electrolytic ions}

The dimensions of the ions constituting both the ILs and the organic electrolyte were evaluated using a computer model (MS Modelling v3.2.0.0) which is a modified version of the Allinger's MM2 force field [22]. For the organic electrolyte solution, the computed value accounts for the non solvated state of the ionic species, given that the number of solvent molecules (solvation shell) is unknown: $\mathrm{TEA}^{+}(0.68 \mathrm{~nm})$ and $\mathrm{BF}_{4}^{-}(0.44 \mathrm{~nm})$. The results of the optimized structures of the ILs showed that the ions present an irregular shape [3] therefore the approximation of the spherical-like geometry to compute the ionic dimensions as radius or diameter is not valid for our systems (particularly the IL). Based on the 3D geometry of the ions in its minimum energy configuration (tetrahedral-like), we have computed the limiting size (ls) 
corresponding to the thickness of the ions that limits the accessibility into the pores, disregarding the maximum distance associated to their length $(L)$. For clarity, a simple scheme of the shape of the ions and the computed dimensions is shown in Figure 1. This approximation provides an estimation of the limiting size of the ions considering rigid structures that could limit the accessibility into the carbon pore network.

As expected, the cation size ls increases with the length of the alkyl chain, following the trend: IL-3 (0.85 nm), IL-4 (0.99 nm), IL-7 (1.35 nm), IL-12 $(1.98 \mathrm{~nm})$. The cations dimensions are much larger than the estimated for the counteranion $\mathrm{Tf}_{2} \mathrm{~N}(0.64 \mathrm{~nm})$ which has an almost spherical-shape comparable to the $\mathrm{TEA}^{+}$and $\mathrm{BF}^{-}$ions from the organic electrolyte.

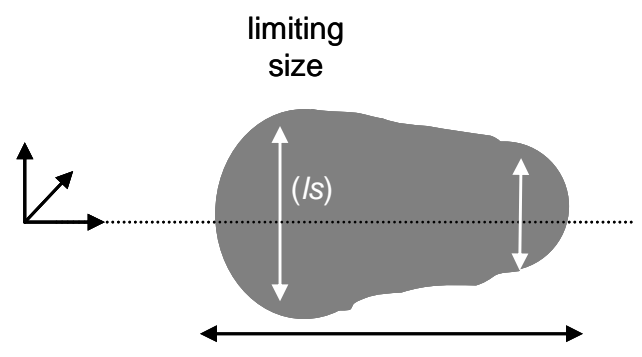

Length $(L)$

Figure 1. Illustration of the irregular shape of the cations in the ionic liquids, showing the computed limiting size (ls). This model may also be used for the $\mathrm{TEA}^{+}$cation, where $l s=L$.

\subsection{Characterization of the carbon materials}

For this study we have selected two pore-controlled carbon materials with very narrow PSD as a tool for exploring the relation between the pore dimensions and those of the electrolyte during the performance of the EC. Their porosity was evaluated by means of gas adsorption. The main nanotextural parameters are compiled in Table 1. The corresponding nitrogen adsorption isotherms and the pore size distributions are shown in Figure 2.

Table 1. Nanotextural characteristics of the porous carbon materials used as electrodes.

\begin{tabular}{lccccc}
\hline Sample & $\begin{array}{c}\mathbf{S}_{\mathbf{D F T}}{ }^{\mathbf{a}} \\
{\left[\mathbf{m}^{\mathbf{2}} \mathbf{g}^{-1} \mathbf{]}\right.}\end{array}$ & $\begin{array}{c}\mathbf{V}_{\text {TOTAL }}^{\mathbf{a}} \\
{\left[\mathbf{c m}^{\mathbf{3}} \mathbf{g}^{-\mathbf{1}} \mathbf{]}\right.}\end{array}$ & $\begin{array}{c}\mathbf{V}_{\text {micro }} \mathbf{b} \\
{\left[\mathbf{c m}^{\mathbf{3}} \mathbf{g}^{-\mathbf{1}} \mathbf{]}\right.}\end{array}$ & $\begin{array}{c}\mathbf{V}_{\text {meso }} \mathbf{b} \\
{\left[\mathbf{c m}^{\mathbf{3}} \mathbf{g}^{-\mathbf{1}} \mathbf{]}\right.}\end{array}$ & $\mathbf{V}_{\text {micro }} / \mathbf{V}_{\text {meso }}$ \\
\hline $\mathbf{C C}$ & 968 & 0.432 & 0.318 & 0.033 & 9.7 \\
$\mathbf{Z 4}$ & 1991 & 1.636 & 1.150 & 0.296 & 3.9 \\
\hline
\end{tabular}

a evaluated at relative pressure $\mathrm{p} / \mathrm{po}=0.95$ in the $\mathrm{N}_{2}$ adsorption isotherms at $-196^{\circ} \mathrm{C}$

${ }^{\mathrm{b}}$ evaluated from the DFT method applied to the $\mathrm{N}_{2}$ adsorption isotherms at $-196^{\circ} \mathrm{C}$

Sample CC exhibits a type I isotherm in the BDDT classification (Brunauer-Deming-Deming-Teller) [23], with an abrupt knee at low relative pressures, 
indicating that it is mainly composed of small size micropores. This is also supported by the fact that the pore volume determined by $\mathrm{CO}_{2}$ adsorption is larger than the corresponding volume of micropores "seen" by $\mathrm{N}_{2}\left(0.318\right.$ vs $0.353 \mathrm{~cm}^{3} \mathrm{~g}^{-1}$ for $\mathrm{N}_{2}$ and $\mathrm{CO}_{2}$, respectively). In contrast, sample Z4 presents a type I-IV hybrid shape isotherm, characteristic of materials with a welldeveloped mesoporosity.

In both cases the micropore volume accounts for almost three quarts of the total pore volume, notwithstanding their pore size distributions (PSD) and total pore volumes are rather different. This is clearly seen in Figure 2 B, which depicts the incremental pore volumes vs pore width (w) calculated by the QSDFT theory [21]. Whereas CC carbon has most of the pores below $1 \mathrm{~nm}$ (maximum of the QSDFT model is centred at ca. $0.6 \mathrm{~nm}$ ), the carbon obtained from nanocasting of zeolite -Z4- displays a maximum centred at $1 \mathrm{~nm}$, as well as a noticeable contribution of mesopores. As the thickness of the zeolite walls (Na-Y) is around $1 \mathrm{~nm}$ [24], this demonstrates that the replication of the zeolite has been successful since the carbon material has inherited the features of the inorganic scaffold (i.e, porosity, structural periodicity -not shown-). It should be noted that the contribution of the so-called ultramicroporosity (pores smaller than $0.7 \mathrm{~nm}$ ) in $\mathrm{Z} 4$ sample is almost negligible.
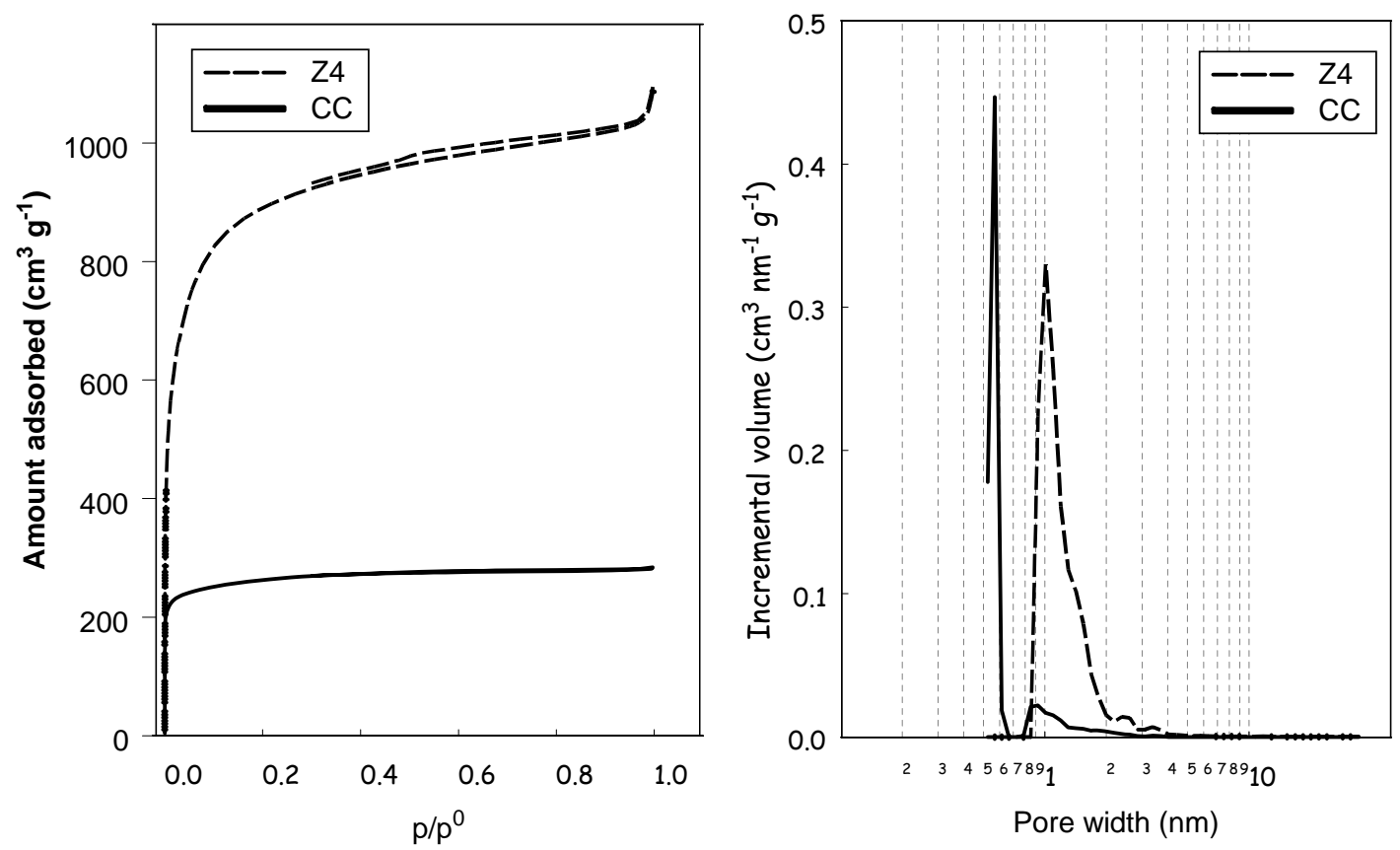

Figure 2. A) $\mathrm{N}_{2}$ adsorption isotherms of the commercial and template carbons and B) pore size distribution evaluated by the QSDFT method applied to the $\mathrm{N}_{2}$ adsorption data.

Nevertheless, it should be mentioned that the location of the maximum in the PSD of such template carbons has been the subject of controversy, since previous values reported in the literature using a non linear DFT model (ca. $1.2 \mathrm{~nm}$ ) for carbons prepared following a similar 
nanocasting route are above the expected value $[19,24,25]$. In this work, we have used the improved QSDFT model for computing the gas adsorption data, and since the result obtained matches with the expected value of $1 \mathrm{~nm}$ based on crystallographic data for zeolites (i.e., zeolite walls bring about the pores of the template carbon), we can assume that the QSDFT model provides a reasonable description of the pore-controlled carbon.

Both CC and Z4 carbons display a sharp PSD as opposed to the typically broad one in carbons obtained from conventional activation methods. Such feature makes them ideal electrode materials for investigating the role of porosity and ions accessibility during the operation of an EDLC. It is also noteworthy that the average pore size of these two carbons is just below or above the recently accepted optimal pore size for a conventional electrolyte and an ionic liquid $[9,26]$.

\subsection{Electrochemical performance of the capacitors}

According to the dimensions of the ionic species and the nanotextural parameters of the carbons derived from gas adsorption data (Table 1), for all the electrolytes the anions should be able to enter in most of pores, not being this the case of the cationic species. The dimensions of the anions in the ionic liquids and in the organic electrolyte are much smaller than those of their corresponding countercations. Therefore, taking into account the series dependence of the overall capacitance of a two-electrode capacitor [2], we can assume that this value is controlled by the electrode with the smallest capacitance, i.e., where the cations are sorbed. With enlarging the alkyl chain in the ILs, one could expect to observe the effect of accessibility restrictions to the porous features of the electrode material in the form of capacitance changes.

Figure 3 shows the cyclic voltammograms - $\mathrm{CV}$ - of the capacitors built in the different electrolytes (tetraethylammonium tetrafluoroborate in acetonitrile and the solvent free ILs) at room temperature. The corresponding values of capacitances are given in Table 2 .

Table 2. Gravimetric capacitance of the EDL capacitors built with the pore controlled carbons $\mathrm{Z} 4$ and $\mathrm{CC}$ - for the different electrolytes up to $1 \mathrm{~V}$ of voltage range

\begin{tabular}{llccccc}
\hline & TEABF $_{\mathbf{4}}$ & IL-3 & IL-4 & IL-7 & IL-12 \\
\hline & Limiting size $(l s)[\mathrm{nm}]$ & 0.68 & 0.85 & 0.99 & 1.35 & 1.98 \\
\hline \multirow{4}{*}{$\mathbf{Z 4}$} & Specific Capacitance $\left[\mathrm{F} \mathrm{g}^{-1}\right]$ & 96 & 84 & 83 & 55 & 41 \\
& Normalized Capacitance $\left[\mu \mathrm{F} \mathrm{cm}^{-2}\right]$ & 4.8 & 4.2 & 4.1 & 2.8 & 2.1 \\
& $\mathrm{~V}_{\text {accessible }}\left[\mathrm{cm}^{3} \mathrm{~g}^{-1}\right]$ & 1.626 & 1.626 & 1.460 & 0.685 & 0.338 \\
& $\% \mathrm{~V}_{\text {accessible }}$ & 99 & 99 & 89 & 42 & 21 \\
\hline \multirow{3}{*}{ Specific Capacitance $\left[\mathrm{F} \mathrm{g}^{-1}\right]$} & 92 & 2 & - & - & - \\
& Normalized Capacitance $\left[\mu \mathrm{F} \mathrm{cm}^{-2}\right]$ & 9.5 & 0.2 & - & - & - \\
$\mathbf{C C}$ & $\mathrm{V}_{\text {accessible }}\left[\mathrm{cm}^{3} \mathrm{~g}^{-1}\right]$ & 0.160 & 0.160 & 0.129 & 0.084 & 0.051 \\
& $\% \mathrm{~V}_{\text {accessible }}$ & 37 & 37 & 30 & 20 & 12 \\
\hline
\end{tabular}


When pure ionic liquids are used as electrolytes, we observe significant differences between the two carbons even in the case of the IL3, which cationic limiting size is the smallest in the series of ionic liquids. The counteranion is the same for al the ILs; its dimensions are smaller that the cationic species and comparable to the $\mathrm{TEA}^{+}$and $\mathrm{BF}_{4}{ }^{-}$ions from the organic electrolyte. In this case, the electrochemical device showed a negligible value of capacitance for $\mathrm{CC}$ carbon $\left(2 \mathrm{~F} \mathrm{~g}^{-1}\right)$, demonstrating that IL-3 cation is too big for the pores of the carbon cloth. For the template Z4 carbon, the capacitance values decrease gradually for the bulky ionic liquids, showing a dependence with the limiting size of the cations. This correlation is in good agreement with previous findings using a commercial activated carbon [3].

Besides the expected trend of the capacitance with the size of the ionic liquids, one striking result is that the two carbon samples present somewhat similar values of specific gravimetric capacitance in the organic electrolyte, in spite of their large differences in porosity. Z4 carbon having a total pore volume about 4 times larger than that of $\mathrm{CC}$ carbon. Such effect is more remarkable if the specific capacitance is normalized per specific surface unit $\left(\mu \mathrm{F} \mathrm{cm}{ }^{-2}\right)$, where that of CC carbon is almost twice higher than for the template carbon (Table 2).
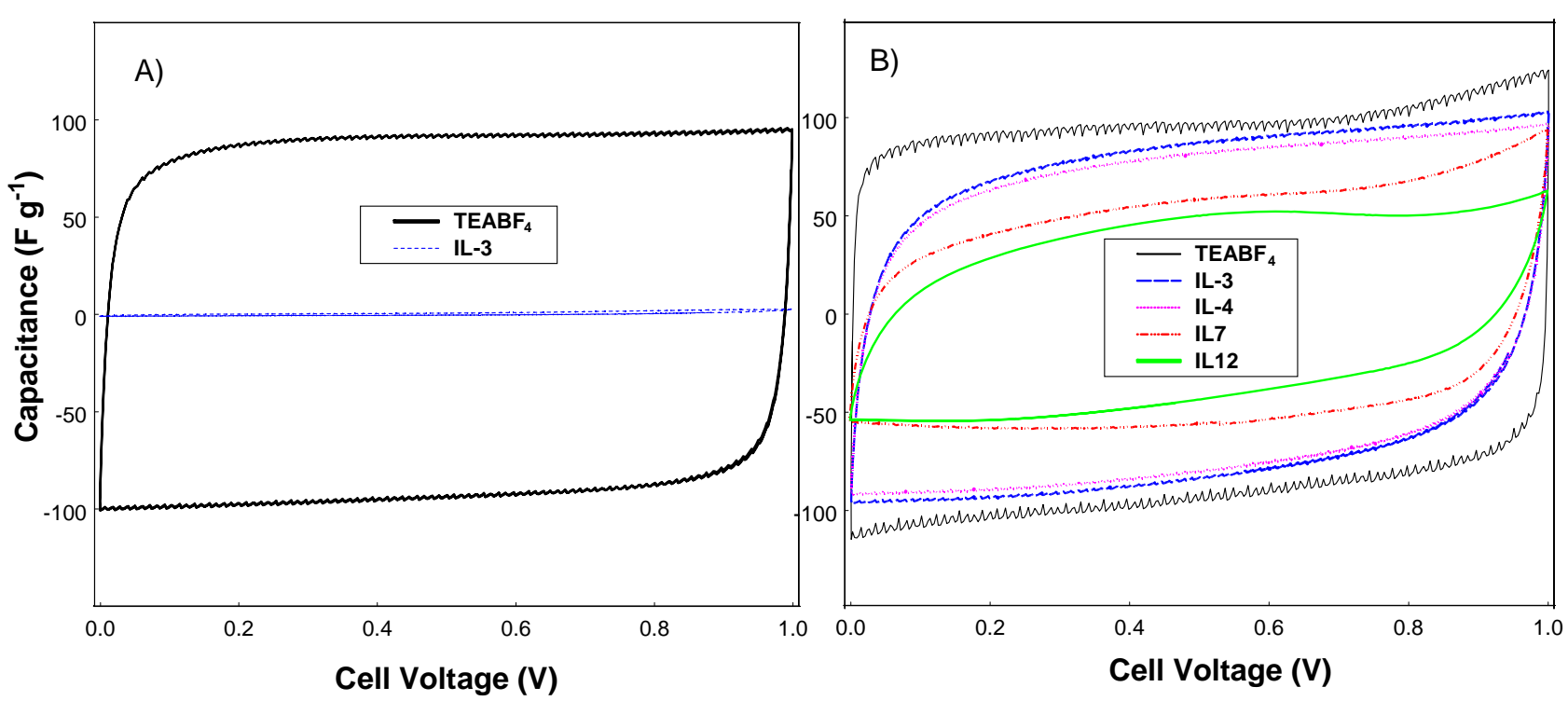

Figure 3. Cyclic voltammograms of the pore controlled carbons at $1 \mathrm{mV} \mathrm{s}^{-1}$ in the different electrolytes. A) carbon cloth $\mathrm{CC}, \mathrm{B})$ template $\mathrm{Z} 4$ carbon.

Which is more surprising is that according to the dimensions calculated for the non solvated ionic species of $\mathrm{TEABF}_{4}$ (Table 2) and the pore widths evaluated by gas adsorption data (Figure $2 \mathrm{~B}$ ), $90 \%$ of the porosity of CC carbon is below the computed cation size (i.e., $0.68 \mathrm{~nm}$ for $\left.\mathrm{TEA}^{+}\right)$. Therefore it would have been reasonable to expect a low capacitance for this carbon in the organic electrolyte, due to restricted accessibility of the cations. Given that similar values have been reported in the literature for the dimensions of $\mathrm{TEABF}_{4}[27,28$ and references therein 
reported] using several methods, and that QSDFT reproduces faithfully the experimental gas adsorption data for this carbon, we neglect miscalculations or experimental errors.

Such unexpected result concerning the electrochemical features of CC carbon confirms that the cationic species enter desolvated in the pores. Moreover, under the effect of the electric field imposed by the polarization of the electrodes, they enter distorted in the pores and showing slightly lower dimensions that their computed limiting size (ls). It appears then that the approximation of computing the ionic dimensions based on their rigid and optimized minimum energy structure does not apply when the ions are forced to move driven by the imposed polarization of the electrodes. In fact, we have tried to estimate the dimensions of the distorted ions, by computing several conformations and we have obtained that the limiting size (ls) in the case of $\mathrm{TEA}^{+}$cation may apparently go down to values below the average pore size of CC carbon corresponding to the maximum value in its PSD (Figure 2B). Moreover, since the applied voltage is limited to 1 Vas above-mentioned, any contribution due to ions intercalation can be discarded. For the case of quaternary ammonium $\mathrm{R} 4 \mathrm{~N}+$ cations and both porous and graphitized nonporous carbons, intercalation has been observed at voltage higher than $2.5 \mathrm{~V}$ [16-18].

On the other hand, still the pore volume of $\mathrm{Z} 4$ is far much larger than that of $\mathrm{CC}$ carbon whereas the specific gravimetric capacitance obtained in the organic electrolyte is rather similar for the two carbons. Such behaviour must be interpreted in terms of the differences in the pore size. Indeed, calculation of the accessible pore volume as the volume fraction of pores larger than the cation computed size (i.e., $0.68 \mathrm{~nm}$ ) by applying the QSDFT model to the nitrogen adsorption data, confirms that above $99 \%$ of the overall porosity of Z4 carbon is accessible for the $\mathrm{TEA}^{+}$cation (above $0.68 \mathrm{~nm}$ ). Comparatively, only about $37 \%$ of the pores in $\mathrm{CC}$ carbon were larger than $0.68 \mathrm{~nm}$ (non distorted limiting dimension of the cation). It appears that the pores in Z4 carbon are too wide for an effective interaction of ions with the pore walls and consequently a large fraction of the pore volume is underused. Consequently, the distance (d) between the electrode surface and the ions increases and therefore the capacitance falls, according to Eq. (1):

$$
\mathrm{C}={ }_{\varepsilon} \mathrm{S} / \mathrm{d}
$$

In the case of CC sample, its relatively high capacitance value is due to the optimal match between the pore size and the dimensions of this electrolyte, especially when distorted. This situation gives rise to an enhanced capacitance due to multiple interactions of the ions with the pore walls of the carbon cloth (Figure 4).

It should be pointed out then that when distorted ions enter the pores which size is smaller that the limiting size of the ionic species of the electrolyte, such confinement state does not allow the 
formation of an electric double layer; the ions would rather form a monolayer surrounded by the charged carbon surfaces. A model where ions form an electric wire-in-cylinder inside micropores of small sizes has recently been proposed by Huang et al. [29].

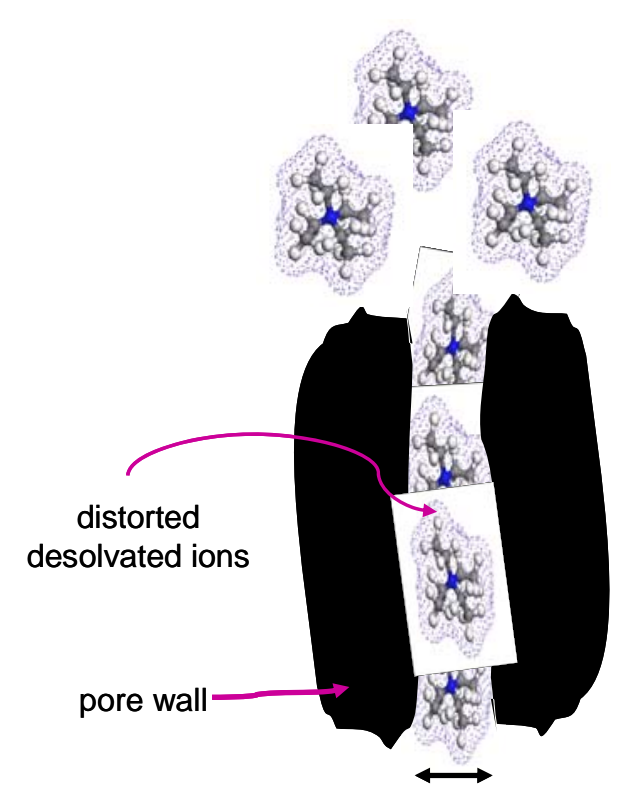

Pore width $0.6 \mathrm{~nm}$

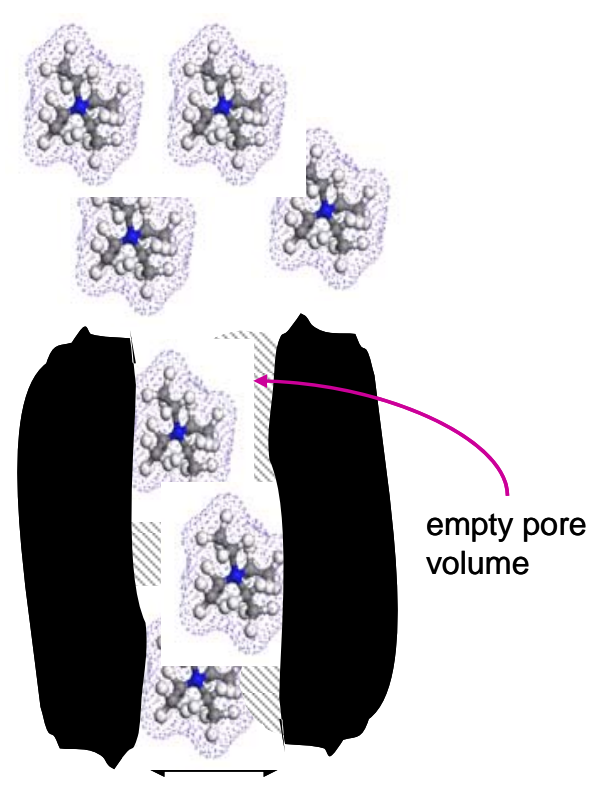

Pore width $1 \mathrm{~nm}$

Figure 4. Illustration of the pore-to-ion interactions inside the pores of carbon electrodes; A) pore width matching the dimensions of distorted electrolyte cations -CC in organic medium-, and B) pore width much larger than the electrolyte limiting size, ls -Z4 in organic medium-.

For corroborating the accessibility of the ions during the operation of the capacitor, we recorded separately the potential range of each individual electrode (positive and negative) vs a silver wire pseudo-reference electrode, while charging/discharging the capacitor up to a given voltage. In such configuration we can obtain the real working potential window of each electrode. Taking into account that charges at the positive $\left(\mathrm{q}^{+}\right)$and the negative $\left(\mathrm{q}^{-}\right)$electrodes have to be balanced, and that there is an excess of electrolyte in our system, the charge balance should follow the equation $\mathrm{d} q^{+}=\mathrm{d} q^{-}$. The charge stored by each electrode depends on the specific capacitance $(C)$, the potential range for the charge/discharge process $(\Delta E)$, and the mass of the electrode $(m)$ according to:

$d q=C \Delta E m$

and then

$m^{+} C^{+} \Delta E^{+}=m^{-} C^{-} \Delta E^{-}$

Since we use the same mass of electrodes $\left(\mathrm{m}^{+}=\mathrm{m}^{-}\right)$, the relationship becomes: 
$C^{+} \Delta E^{+}=C^{-} \Delta E^{-}$

(eq. 4)

The results corresponding to an applied cell voltage of $0.6 \mathrm{~V}$ and $1 \mathrm{~V}$ in TEABF4 and IL-3 electrolytes are shown in Figure 5. A close look at the plots A in Figure 5 reveals that in organic medium, the potential range in which the negative and the positive electrodes operate is nearly equal, indicating that the cell voltage splits equally between the two electrodes for both carbons $\mathrm{CC}$ and Z4.

According to the equation (4), for a symmetric capacitor with two electrodes having the same mass it indicates that the capacities given by the cation and anion are similar $\left(\mathrm{C}^{+}=\mathrm{C}^{-}\right)$, which seems reasonable if both ions are of close sizes and confirms the accessibility of $\mathrm{TEABF}_{4}$ ions to the porosity of CC carbon electrodes.

Based on Eq. (1) giving the dependence of capacitance with the interphasial area, and assuming that the dielectric constant of the electrolyte is the same for both electrodes, we find that the ratio between the interphasial area and the effective distance for the cation and anion, respectively, are similar $(\mathrm{Sc} / \mathrm{dc}=\mathrm{Sa} / \mathrm{da})$. Given the small size of the anion, we can assume that the anionic distance (da) is smaller that that of the distorted cation (dc). Also, de should be somewhat close to half the average pore size for the carbon cloth (i.e., $0.58 / 2=0.29 \mathrm{~nm}$ ). In such case, the interphasial area Sc occupied by TEA+ is higher than that of BF4, which is in good agreement with the assumed bigger size for TEA+.
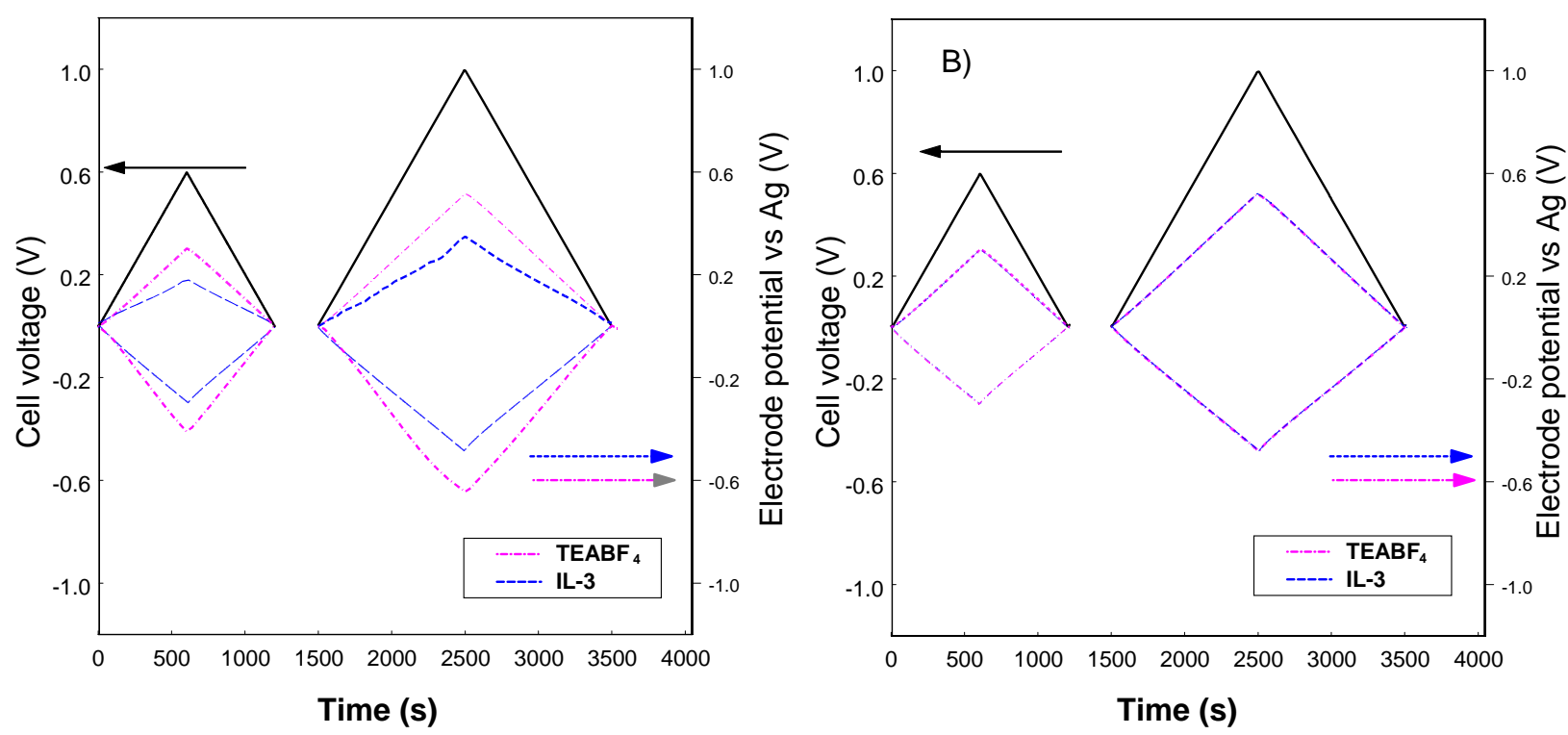

Figure 5. Working potential range of the positive and negative electrodes (dotted and dashed lines read in right $\mathrm{Y}$ axis) for two voltage ranges $(0.6$ and $1 \mathrm{~V}$; solid lines read in left $\mathrm{Y}$ axis) in two different electrolytes for the carbon cloth (A) and template carbon (B). 
Extending this study to the series of ionic liquids, we observed low values of capacitance for the carbon cloth (CC) in IL-3 and IL-4. Only few cations enter in the pores of the carbon cloth which are compatible with the limiting size of IL-3 and IL-4. Although such cells are not realistic for practical application, it was possible to record the real potential range of each electrode during the polarization of the capacitor (Figs. 5 and 6).

As opposed to TEABF4 data, when using the ionic liquids IL-3 and IL-4 in presence of CC, the potential range of the positive electrode $(\mathrm{DE}+)$ becomes smaller than that of the negative electrode (DE $+<\mathrm{DE} \_$). Moreover, as the cationic limiting size (ls) increases, the differences in the working potential window of the negative and the positive electrodes become larger. Such behaviour indicates that the capacitance values are affected by cationic restrictions to the pores. Taking into account the nanotextural data obtained from gas adsorption on $\mathrm{CC}$, it seems reasonable to obtain such effect in the carbon cloth which possesses a narrow distribution of pores of small size. It appears that bulky ionic liquids (IL-3 and IL-4) cannot accommodate their sizes so as to enter in the narrow micropores of the carbon cloth. Only those pores which size is compatible with the limiting cationic size of IL-3 and IL-4 are accessible; consequently, the effective cationic thickness dc increases and thus according to Eq. (1) the capacitance of the negative electrode decreases. Likewise, given the small size of the counteranion - which can access to pores of smaller sizes - the anionic distance is expected to be smaller than the cationic one $(\mathrm{da}<\mathrm{dc})$ and thus $\mathrm{C}+$ would be larger than $\mathrm{C}_{-}$. Considering Eq. (4), this turns out in DE $+<$ DE_, which is in good agreement with our experimental results (Figs. 5 and 6).

By contrast, in the case of the Z4 carbon, such behaviour is only observed when IL-12 is used as electrolyte (Figs. 5 and 6). According to gas adsorption data (Fig. 2B), most of the pores of this carbon are $1 \mathrm{~nm}$ size, above the limiting size of TEABF4, IL-3 and IL-4 cations; therefore no accessibility restrictions apply. On the contrary, when the limiting size of the cation is further increased (IL-12), again the broadening of the potential range for the negative electrode is observed ( $\mathrm{DE}+<\mathrm{DE})$. This indicates that the bulky IL-12 cation is not able to sufficiently distort for adjusting its dimensions to the pore sizes, and only a fraction of the porosity is accessible to this cation (i.e., the biggest pores). As abovementioned, this brings about in an increase in the cationic distance which, according to Eq. (1), is responsible for the fall in capacitance of the negative electrode $\left(\mathrm{C}_{-}\right)$. In this situation, the potential range of the later electrode is enlarged, in order to counterbalance the loss in capacitance and to maintain the total charge balance in the system. For the same reason, the potential window of the electrode where the anions are sorbed - where it is assumed that there are no size restrictions - is shortened. 

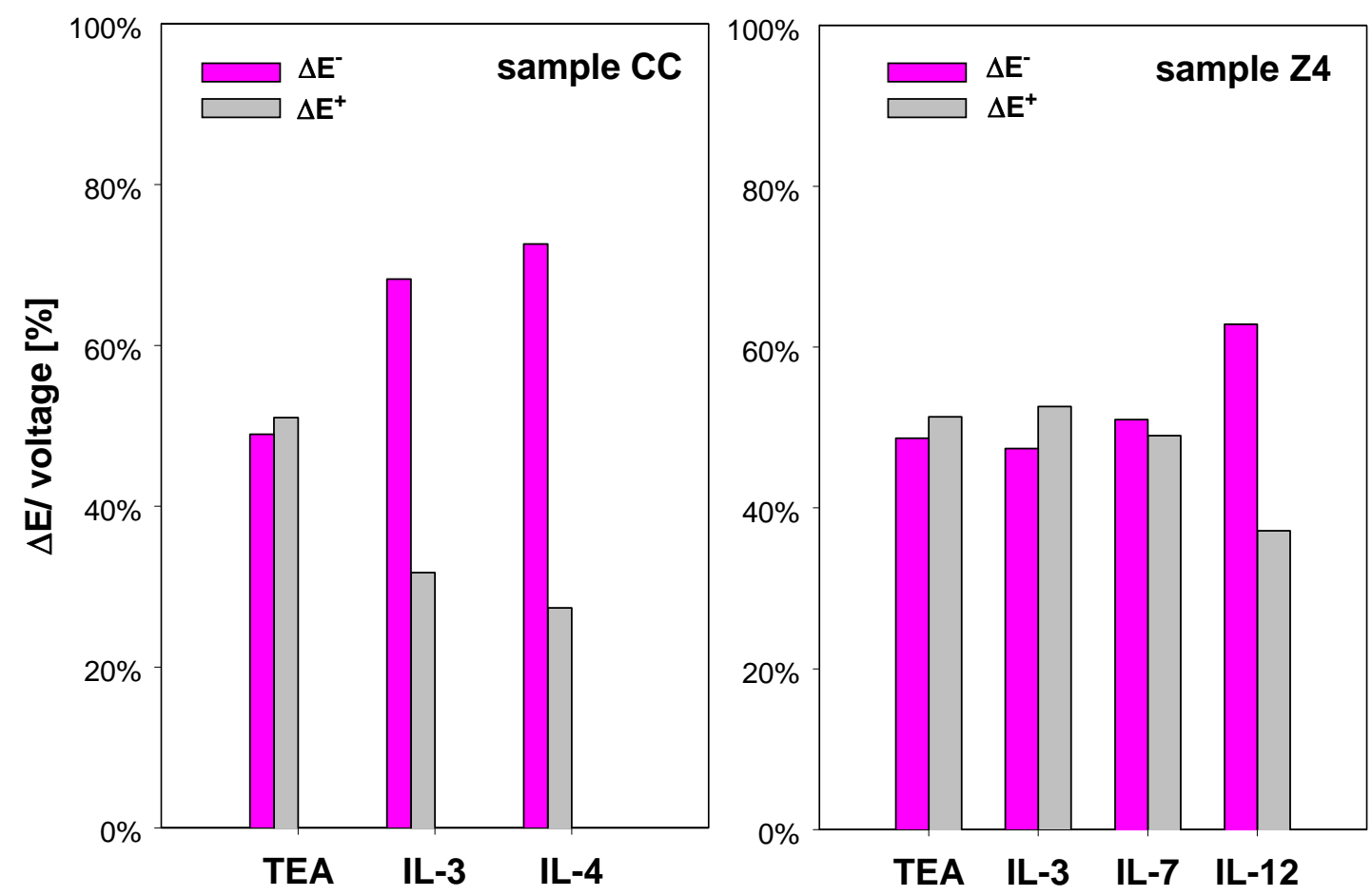

Figure 6. Evolution of the working electrode potential window of the negative and positive electrodes in the different electrolytic media using the carbon cloth (left) and template carbon (right) as electrode materials.

This behaviour is nevertheless not observed in the pore controlled Z4 carbon in IL-3 electrolyte (Figure 6 ) or $\mathrm{TEA}^{+}$, since its average pore size is centred at $1 \mathrm{~nm}$, above the limiting size of these ions, and therefore no accessibility restrictions apply. On the contrary, when the limiting size of the cation is further increased (IL-12), again the broadening of the potential range for the negative electrode is observed. This indicates that while IL-7 would be able to distort to adjust its dimensions to the pore sizes, this is not the case in a much larger cation (IL-12).

\section{Conclusions}

The utilization of porous carbons with a narrow micropore size distribution has allowed the confinement state of the ionic species inside the pores of carbon electrodes to be investigated during the performance of electrochemical capacitors. Conventional organic medium and solvent-free ionic liquids have been explored as electrolytes. Our results confirm what has been 
suggested in the literature about the desolvated state of ions inside the porosity of carbon electrodes. Moreover, we have demonstrated that under the effect of the electric field imposed by the polarization of the electrode,the ions are flexible and their configuration (and limiting size) can be in situ modified so as to squeeze into the electrode pores. This behaviour does not apply systematically for all the electrolytes tested, as it depends on the flexibility of the ions given by their geometry - and on the relationship between the pore size and the ions dimension, so that the distortion is restricted for bulky ions which limiting size is larger than the pore size. The distortion of the ionic species changes the confinement state inside the pores, provoking that the electrosorbed ions form a monolayer close to the pore walls of the electrode material instead of the usual electric double-layer where no restrictions apply.

\section{Acknowledgements}

The authors thank Actitex (France) for kindly supplying the carbon cloth. COA thanks her Ramon y Cajal Research Contract and the Spanish-French Collaborative Grant (Accion Integrada HF2006-0115) for supporting her stay at CRMD.

\section{References}

[1] Conway BE. Electrochemical Supercapacitors, Kluwer Academic Plenum, New York, 1999.

[2] Frackowiak E, Béguin F. Carbon materials for the electrochemical storage of energy in capacitors. Carbon 2001; 39:937-950.

[3] Ania CO, Pernak J, Stefaniak F, Raymundo-Piñero E, Béguin F. Solvent-free ionic liquids as in situ probes for assessing the effect of ion size on the performance of electrical double layer capacitors. Carbon 2006;44:3113-3126.

[4] Raymundo-Piñero E, Kierzek K, Machnikowski J, Béguin F. Relationship between the nanoporous texture of activated carbons and their capacitance properties in different electrolytes. Carbon 2006;44:2498-2507.

[5] Salitra G, Soffer A, Eliad L, Cohen Y, Aurbach D. Carbon electrodes for double-layer capacitors. I. Relations between ion and pore dimensions. J Electrochem Soc 2000;147:2486-2493.

[6] Chmiola J, Yushin G, Dash R, Gogotsi Y. Effect of pore size and surface area of carbide derived carbons on specific capacitance. J Power Sources 2006;158:765-772.

[7] Eliad L, Pollak E, Levy N, Salitra G, Soffer A, Aurbach D. Assessing optimal pore-toion size relations in the design of porous poly(vinylidene chloride) carbons for EDL capacitors. Appl Phys A 2006;82:607-613. 
[8] Eliad L, Salitra G, Soffer A, Aurbach D. Ion sieving effects in the electrical double layer of porous carbon electrodes: estimating effective ion size in electrolytic solutions. J Phys Chem B 2001;105:6880-7.

[9] Largeot C, Portet C, Chmiola J, Taberna P, Gogotsi Y, Simon P. Relation between the ion size and pore size for an electric double layer capacitor. J. Am. Chem . Soc., 130 (2008) 2730-2731.

[10] Lee J, Kim J, Hyeon T. Recent progress in the synthesis of porous carbon materials. Adv Mater 2006;18:2073-2094.

[11] Hiroyuki O, editor. Electrochemical Aspects of Ionic Liquids. Wiley-Interscience, New York, 2005.

[12] Ue M, Takeda M, Toriumi A, Kominato A, Hagiwara R, Ito Y. Application of LowViscosity Ionic Liquid to the Electrolyte of Double-Layer Capacitors. J Electrochem Soc 2003;150:A499-502.

[13] Frackowiak E, Lota G, Pernak J. Room-temperature phosphonium ionic liquids for supercapacitor applications. J Appl Phys Lett 2005;86:164104-1-164104-3.

[14] Balducci A, Dugas R, Taberna PL, Simon P, Plee D, Mastragostino M, Passerini S. High temperature carbon-carbon supercapacitor using ionic liquid as electrolyte. J Power Sources 2007;165:922-927.

[15] Lazzari M, Mastragostino M, Soavi F. Capacitance response of carbons in solvent-free ionic liquid electrolytes. Electrochem Comm 2007;9:1567-1572.

[16] Berthelot J, Simonet J. Le graphite HOPG: des comportements anodiques et cathodiques particuliers. Electrochim Acta 1984;29:1181-6.

[17] Takeuchi M, Koike K, Maruyama T, Mogami A, Okamura M. Electrochemical intercalation of tetraethylammonium tetrafluoroborate into $\mathrm{KOH}$-treated carbon consisting of multi-graphene sheets for an electric double layer capacitor. Electrochemistry 1998;66:1311-7.

[18] Hahn M, Barbieri O, Campana FP, Kotz R, Gallay R. Carbon based double layer capacitors with aprotic electrolyte solutions: the possible role of intercalation/insertion processes. Appl Phys A 2006;82:633-8.

[19] C.O. Ania, V. Khomenko, E. Raymundo-Piñero, J.B. Parra, F. Béguin, The Large Electrochemical Capacitance of Microporous Doped Carbon Obtained by Using a Zeolite Template. Adv Funct Mat 2007;17:1828-1836.

[20] Pernak J, Stefaniak F, Węglewski J. Phosphonium acesulfamate based ionic liquids. Eur J Org Chem 2005;4:650-652.

[21] Ravikovitch P, Neimark AV. Density Functional Theory Model of Adsorption on Amorphous and Microporous Silica Materials. Langmuir 2006;22:11171-11179. 
[22] Kao J, Allinger NL. Conformational analysis. 122. Heats of formation of conjugated hydrocarbons by the force field method. J Am Chem Soc 1977;99:975-986.

[23] Brunauer B, Deming LS, Deming WE, Teller E. On a Theory of the van der Waals Adsorption of Gases. J Am Chem Soc 1940;62:1723-1732.

[24] Matsuoka K, Yamagishi Y, Yamazaki T, Setoyama N, Tomita A, Kyotani T. Extremely high microporosity and sharp pore size distribution of a large surface area carbon prepared in the nanochannels of zeolite Y. Carbon 2005;43:876-879.

[25] Roussel T, Jagiello J, Pellenq RJM, Thommes M, Bichara C. Testing the feasibility of using the Density Functional Theory route for pore size distribution calculations of ordered microporous carbons. Mol Simulation 2006;32:551-555.

[26] Chmiola J, Largeot C, Taberna PL, Simon P, Gogotsi Y. Desolvation of ions in subnanometer pores and its effect on capacitance and double layer theory. Angew Chemie 2008;47:1-5.

[27] Kim YJ, Horie Y, Ozaki S, Matsuzawa Y, Suezaki H, Kim C, Miyashita N, Endo M. Correlation between the pore and solvated ion size on capacitance uptake of PVDCbased carbons. Carbon 2004;42:1491-1500.

[28] Ue M. Mobility and ionic association of lithium quaternay ammonium slats in propylene carbonate and g-butyrolactone, J Electrochem Soc 1994;141(12):3336-3341.

[29] Huang J, Sumpter BG, Meunier V. A universal model for nanoporous carbon supercapacitors applicable to diverse pore regimes, carbon materials and electrolytes. Chem Eur J 2008;14:6614-6626. 